\title{
An adaptive bolus calculator to minimize the impact of inaccurate insulin to carbohydrate ratio
}

Cite as: AIP Conference Proceedings 2293, 260008 (2020); https://doi.org/10.1063/5.0026578

Published Online: 25 November 2020

Francisco Miranda, Carlos Abreu, and Paula Felgueiras

. Export citation

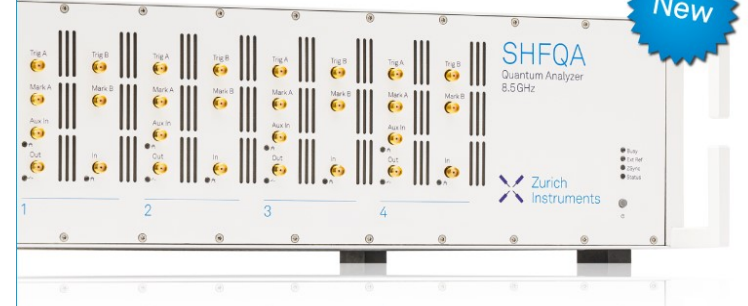

Your Qubits. Measured.

Meet the next generation of quantum analyzers

- Readout for up to 64 qubits

- Operation at up to $8.5 \mathrm{GHz}$,

Find out more

mixer-calibration-free

- Signal optimization with minimal latency 


\title{
An Adaptive Bolus Calculator to Minimize the Impact of Inaccurate Insulin to Carbohydrate Ratio
}

\author{
Francisco Miranda ${ }^{1,2}$, Carlos Abreu $^{1, a)}$ and Paula Felgueiras ${ }^{1,3}$ \\ ${ }^{1}$ Instituto Politécnico de Viana do Castelo, Viana do Castelo, Portugal \\ ${ }^{2}$ CIDMA, Universidade de Aveiro, Aveiro, Portugal \\ ${ }^{3}$ Unidade Local de Saúde do Alto Minho, Hospital de Santa Luzia, Viana do Castelo, Portugal \\ ${ }^{a)}$ Corresponding author: cabreu@estg.ipvc.pt
}

\begin{abstract}
Patients with type 1 diabetes mellitus use intensive insulin therapy to suppress their insulin needs and avoid the adverse consequences of chronic hyperglycemia. Intensive insulin therapy consists of a combination of basal insulin and bolus insulin. While the basal insulin dose is periodically adjusted in collaboration with the healthcare team, patients have to estimate the bolus insulin dose by themselves, before each meal. To accurately estimate the bolus insulin dose, patients must know the carbohydrates content of each meal and their insulin to carbohydrate ratio. The insulin to carbohydrate ratio is initially calculated by experienced diabetologists using high-quality data. However, regarding the glucose complex metabolism, it varies over the day due to several factors. Consequently, daily, patients use approximate values to estimate their bolus insulin. Thus, depending on the error of the insulin to carbohydrate ratio estimates, the patient could experience hypo or hyperglycemic events. Therefore, to avoid the consequences of inaccurate bolus insulin and to improve the patient's glycemic control, this work presents an adaptive insulin bolus calculator that uses the patient's glycemic data to dynamically adjust the mealtime bolus and compensate for the adverse effects of inaccurate insulin to carbohydrate ratio estimates.
\end{abstract}

\section{INTRODUCTION}

Type 1 diabetes mellitus $(T 1 D M)$ is a chronic disease characterized by the autoimmune destruction of insulin-secreting pancreatic $\beta$ cells. Consequently, T1DM patients must supply their bodies with insulin to avoid the deleterious consequences of chronic hyperglycemia. Insulin therapy consists of a basal-bolus regimen, in which the basal insulin is slow-acting insulin that regulates the blood glucose levels between meals, and the bolus insulin is fast-acting insulin to get the postprandial blood glucose on target, according to the carbohydrate intake of each meal [1]. However, to be effective, the bolus insulin dose must be accurate. Although, to correctly calculating it is not simple since it depends on several factors, i.e., the carbohydrate intake at each meal $(\mathrm{CHO})[2,3]$, the Insulin to Carbohydrate Ratio (ICR), the Insulin Sensitivity Factor (ISF), the preprandial blood glucose [4], the postprandial blood glucose target and the insulin remaining active from the last boluses. Concerning the ICR and IS F factors, they are correlated and dynamic [5-7], changing along the day due to several factors, namely, physical activity, stress, health condition, or the hormone cycle, making it hard to get [8]. Indeed, in their daily life, patients use ICR estimates to determine the prandial insulin. So, depending on the magnitude of the error present in the ICR estimates, it could result in off-target postprandial blood glucose levels, or even worse, in hypo or hyperglycemic events. Therefore, it is of great importance to developing methods to minimize the adverse consequences of inaccurate ICR estimates. In this regard, this work introduces an adaptive bolus calculator that uses both on-demand and retrospective data to dynamically adjust the mealtime insulin. On-demand data refers to the patient's blood glucose and carbohydrates intake at mealtime. On the other hand, retrospective data relates to the postprandial blood glucose of previous meals. The flexibility of our method allows using different algorithms and artificial intelligence techniques to compute retrospective data to get the adjusted bolus and, consequently, on-target blood glucose levels. The remaining of this paper presents the proposed method along with an application example. Finally, some future directions are pointed out regarding the assessment and the practical application of the proposed bolus calculator.

International Conference of Numerical Analysis and Applied Mathematics ICNAAM 2019

AIP Conf. Proc. 2293, 260008-1-260008-4; https://doi.org/10.1063/5.0026578

Published by AIP Publishing. 978-0-7354-4025-8/\$30.00 


\section{MATHEMATICAL METHODS}

Consider the following equation:

$$
B=\frac{C H O}{I C R}+\frac{G-G_{T}}{I S F}-I O B,
$$

that is used for patients to determine the prandial and correction boluses to be administrated before each meal, where $B[\mathrm{U}]$ is the bolus insulin, $C H O[\mathrm{~g}]$ are the carbohydrates consumed in the meal, $G[\mathrm{mg} / \mathrm{dL}]$ is the preprandial blood glucose, $G_{T}[\mathrm{mg} / \mathrm{dL}]$ is the blood glucose target, $I O B[\mathrm{U}]$ is the Insulin-on-Board, i.e., it is the insulin remaining active from the previously administrated bolus, and $I C R[\mathrm{~g} / \mathrm{U}]$ and $I S F[\mathrm{mg} / \mathrm{dL} / \mathrm{U}]$ are the insulin to carbohydrate ratio and the insulin sensitivity factor, respectively $[9,10]$.

From the works [5-7], we can consider that $I S F=\alpha \cdot I C R / W$, where $W[\mathrm{~kg}]$ is the patient's bodyweight and $\alpha\left[\mathrm{dL}^{-1}\right]$ is a nonzero positive constant. So, Equation 1 can be rewritten as $B=C H O / I C R+W \cdot\left(G-G_{T}\right) /(\alpha \cdot I C R)-I O B$. In the following analysis, we consider that $I C R$ is properly estimated by a diabetologist and denoted by $I \hat{C} R$, and the $C H O, W, G$, and $I O B$ are correct values.

Let $\delta_{I C R}$ be the $I C R$ estimation error, i.e., $\delta_{I C R}=I C R-I \hat{C} R$. If $\delta_{I C R} \neq 0$ there is an error on $B$ given by $\delta_{B}=B-\hat{B}$, where $\hat{B}=C H O / I \hat{C} R+W \cdot\left(G-G_{T}\right) /(\alpha \cdot I \hat{C} R)-I O B$. Therefore, we have:

$$
\delta_{B}=\frac{\alpha \cdot C H O+W \cdot\left(G-G_{T}\right)}{\alpha \cdot I C R}-\frac{\alpha \cdot C H O+W \cdot\left(G-G_{T}\right)}{\alpha \cdot I \hat{C} R} .
$$

The error in the bolus, $\delta_{B}$, will act as an unplanned correction bolus, and therefore leading to an off-target postprandial blood glucose $\left(G_{\text {postprandial }}\right)$ and an error given by

$$
\delta_{G_{\text {postprandial }}}=G_{\text {postprandial }}-G_{T}=\frac{\delta_{B} \cdot \alpha \cdot I C R}{W} .
$$

As it is easily to prove, $\alpha \cdot C H O+W \cdot\left(G-G_{T}\right)>0$, and replacing $\delta_{B}$ of Equation 3 by Equation 2 for $I C R=I \hat{C} R+\delta_{I C R}$, we obtain:

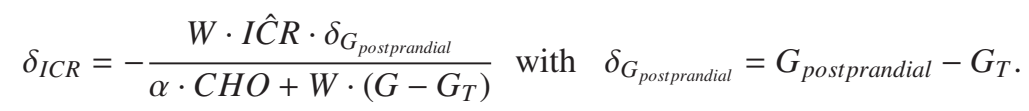

The main purpose of this work is to correct the bolus insulin to minimize the impact of inaccurate insulin to carbohydrate ratio on $G_{\text {postprandial }}$. In this context, we will insert a correction nonzero positive constant $K$ to adjust the estimated bolus to the real bolus, i.e., we search $K$ such that:

$$
\begin{aligned}
& \left(\frac{C H O}{I \hat{C} R}+\frac{W \cdot\left(G-G_{T}\right)}{\alpha \cdot I \hat{C} R}\right) \cdot K-I O B=B \\
\Longleftrightarrow & \frac{\alpha \cdot C H O+W \cdot\left(G-G_{T}\right)}{\alpha \cdot I \hat{C} R} \cdot K=\frac{\alpha \cdot C H O+W \cdot\left(G-G_{T}\right)}{\alpha \cdot I C R} \\
\Longleftrightarrow & K=\frac{I \hat{C} R}{I \hat{C} R+\delta_{I C R}} .
\end{aligned}
$$

Using Equation 4 in Equation 5, we obtain:

$$
\begin{aligned}
& K=\frac{I \hat{C} R}{I \hat{C} R-\frac{W \cdot I \hat{C} R \cdot \delta_{G_{\text {possprantial }}}}{\alpha \cdot C H O+W \cdot\left(G-G_{T}\right)}}
\end{aligned}
$$

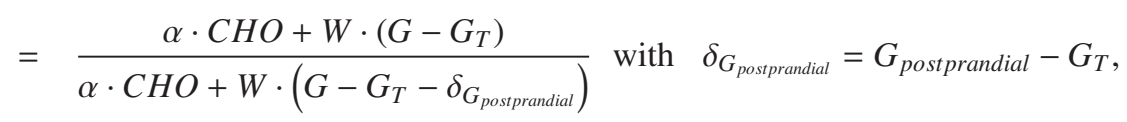


where $G_{\text {postprandial }}{ }^{1}$ is obtained using the method proposed in [11] and $\delta_{G_{\text {postprandial }}}<\left(\alpha \cdot C H O+W \cdot\left(G-G G_{T}\right)\right) / W$. This condition for $\delta_{G_{\text {postprandial }}}$ is necessary to ensure that $\hat{B}>0$. Consider that $\delta_{G_{\text {postprandial }}} \geq(\alpha \cdot C H O+W \cdot(G-G)) / W$. Then, by Equation 3, we obtain that

$$
\begin{aligned}
& \frac{\alpha \cdot C H O+W \cdot\left(G-G_{T}\right)}{W} \leq \frac{(B-\hat{B}) \cdot \alpha \cdot I C R}{W} \\
\Longleftrightarrow & B-\hat{B} \geq \frac{\alpha \cdot C H O+W \cdot\left(G-G_{T}\right)}{\alpha \cdot I C R} \\
\Longleftrightarrow & B-\hat{B} \geq B+I O B \\
\Longleftrightarrow & \hat{B} \leq-I O B \leq 0,
\end{aligned}
$$

that is a contradiction.

\section{APPLICATION EXAMPLE}

Consider a patient with the following characteristics: $I \hat{C} R=11.80 \mathrm{~g} / \mathrm{U}, W=70 \mathrm{~kg}$, and $\alpha=1700 / 6.17 \mathrm{dL}{ }^{-1}[5]$. Suppose that this patient will ingest a meal of $50 \mathrm{~g}$ of carbohydrates, and in the moment of the meal he has $I O B=$ $0.20 \mathrm{U}$ and the preprandial blood glucose, $G$, equal to $110 \mathrm{mg} / \mathrm{dL}$. According to his historical and the method used to calculate an estimate of postprandial blood glucose after the meal, we consider that $G_{\text {postprandial }}=130 \mathrm{mg} / \mathrm{dL}$. Admit that the blood glucose target is $G_{T}=100 \mathrm{mg} / \mathrm{dL}$. So, the error of $G_{\text {postprandial }}$ is $\delta_{G_{\text {postprandial }}}=G_{\text {postprandial }}-G_{T}=$ $30 \mathrm{mg} / \mathrm{dL}<\left(\alpha \cdot C H O+W \cdot\left(G-G_{T}\right)\right) / W \approx 206.80 \mathrm{mg} / \mathrm{dL}$. To minimize the impact of inaccurate insulin to carbohydrate ratio in $G_{\text {postprandial }}$, the patient should correct the bolus insulin using the correction nonzero positive constant $K$ given by Equation 6:

$$
K=\frac{\frac{1700}{6.17} \cdot 50+70 \cdot(110-100)}{\frac{1700}{6.17} \cdot 50+70 \cdot(110-100-30)} \approx 1.17
$$

Therefore, the patient must administer the following corrected bolus insulin:

$$
B_{K}=\left(\frac{C H O}{I \hat{C} R}+\frac{W \cdot\left(G-G_{T}\right)}{\alpha \cdot I \hat{C} R}\right) \cdot K-I O B \approx 5.01 \mathrm{U}
$$

instead of

$$
\hat{B}=\frac{C H O}{I \hat{C} R}+\frac{W \cdot\left(G-G_{T}\right)}{\alpha \cdot I \hat{C} R}-I O B \approx 4.25 \mathrm{U} .
$$

\section{CONCLUSION}

This work presented an adaptive mealtime insulin calculator able to minimize the adverse consequences of incorrect bolus insulin due to inaccurate insulin to carbohydrate ratio estimates. The proposed method allows adjusting the bolus insulin of each meal independently, using meal specific data, either on-demand and retrospective. On-demand data refers to the patients' glycemia and carbohydrates intake at mealtime, on the other hand, retrospective data relates to the postprandial blood glucose of previous meals. The flexibility of our method allows using different algorithms and artificial intelligence techniques to compute retrospective data to get the adjusted bolus and, therefore, improve the patient's glycemic control. Regarding future research activities, we plan to investigate the use of different artificial intelligence techniques to estimate the postprandial blood glucose, $G_{\text {postprandial }}$, and assess the trustworthiness of the adjusting factor $K$. Finally, the proposed method will be assessed by performing several in-silico trials, using the FDA accepted UVa/Padova T1DM Simulator v3.2.

\footnotetext{
${ }^{1} G_{\text {postprandial }}$ is measured two hours after each meal and can be obtained from retrospective data using different techniques. The method proposed in [11] is just an example.
} 


\section{ACKNOWLEDGMENTS}

This work was supported by the Portuguese Foundation for Science and Technology (FCT - Fundação para a Ciência e a Tecnologia), through CIDMA - Center for Research and Development in Mathematics and Applications, within project UID/MAT/04106/2019.

\section{REFERENCES}

[1] 9. Pharmacologic Approaches to Glycemic Treatment: Standards of Medical Care in Diabetes-2019. Diabetes Care, 42(Supplement 1):S90-S102, 2019.

[2] Abreu C., Miranda F., and Felgueiras P. Carbohydrate counting: How accurate should it be to achieve glycemic control in patients on intensive insulin regimens? AIP Conference Proceedings, 2116(1):250009, 2019.

[3] Abreu C., Miranda F., and Felgueiras P. The impact of accurate carbohydrate counting on patient's glycemic targets. In Diabetes Technology \& Therapeutics, volume 21, pages A66-A66. Mary Ann Liebert, Inc., 140 Huguenot Street, 3rd Floor, New Rochelle, NY 10801 USA, 2019.

[4] Abreu C., Miranda F., Dabrowska A., and Felgueiras P. Home glucose meters: How accurate should they be to avoid dysglycemia in patients using carbohydrate counting? AIP Conference Proceedings, 2116(1):250010, 2019.

[5] Davidson P., Hebblewhite H., Steed R., and Bode B. Analysis of guidelines for basal-bolus insulin dosing: Basal insulin, correction factor, and carbohydrate-to-insulin ratio. Endocrine Practice, 14(9):1095-1101, 2008.

[6] Walsh J., Roberts R., and Bailey T. Guidelines for insulin dosing in continuous subcutaneous insulin infusion using new formulas from a retrospective study of individuals with optimal glucose levels. Journal of Diabetes Science and Technology, 4(5):1174-1181, 2010.

[7] Walsh J., Roberts R., and Bailey T. Guidelines for optimal bolus calculator settings in adults. Journal of Diabetes Science and Technology, 5(1):129-135, 2011.

[8] Torrent-Fontbona F., Massana J., and López B. Case-base maintenance of a personalised and adaptive CBR bolus insulin recommender system for type 1 diabetes. Expert Systems with Applications, 121:338-346, 2019.

[9] Walsh J., Roberts R., Bailey T. S., and Heinemann L. Bolus advisors: Sources of error, targets for improvement. Journal of Diabetes Science and Technology, 12(1):190-198, 2018.

[10] Schmidt S. and Nørgaard K. Bolus calculators. Journal of Diabetes Science and Technology, 8(5):1035-1041, 2014.

[11] Abreu C., Miranda F., and Felgueiras P. An adaptive mealtime bolus calculator to minimize the effects of inaccurate carbohydrate counting. AIP Conference Proceedings, 2116(1):250011, 2019. 